\section{Cahiers d'ethnomusicologie}

Anciennement Cahiers de musiques traditionnelles

$18 \mid 2005$

Entre femmes

\title{
Musiques du Yémen. Des hauts plateaux (Sanaa) aux plaines côtières (Tihāma)
}

Yémen : Musiques du cœur de l'Arabie, 2002 ; The Yemen Tihama, Trance \& Dance music from the Red Sea Coast of Arabia, 2002

Samir Mokrani et Luc Weissenberg

\section{OpenEdition}

Journals

Édition électronique

URL : http://journals.openedition.org/ethnomusicologie/406

ISSN : 2235-7688

Éditeur

ADEM - Ateliers d'ethnomusicologie

Édition imprimée

Date de publication : 31 décembre 2005

Pagination : $322-327$

ISSN : 1662-372X

Référence électronique

Samir Mokrani et Luc Weissenberg, « Musiques du Yémen. Des hauts plateaux (Sanaa) aux plaines côtières (Tihāma) », Cahiers d'ethnomusicologie [En ligne], 18| 2005, mis en ligne le 14 janvier 2012 , consulté le 02 mai 2019. URL : http://journals.openedition.org/ethnomusicologie/406

Ce document a été généré automatiquement le 2 mai 2019.

Tous droits réservés 


\section{Musiques du Yémen. Des hauts plateaux (Sanaa) aux plaines côtières (Tihāma)}

Yémen : Musiques du cœur de l'Arabie, 2002 ; The Yemen Tihama, Trance \& Dance music from the Red Sea Coast of Arabia, 2002

\section{Samir Mokrani et Luc Weissenberg}

\section{RÉFÉRENCE}

Musiques du Yémen. Des hauts plateaux (Sanaa) aux plaines côtières (Tihāma) - Yémen : Musiques du cœur de l'Arabie, CD Buda Records, 1978302, 2002.Enregistrements : JeanChristophe Girard, photos et texte : Henri Lecomte. - The Yemen Tihama, Trance \& Dance music from the Red Sea Coast of Arabia, CD Topic Records/British Library TSCD920, 2002. Enregistrements et texte : Anderson Bakewell. Photos : Alain Saint-Hilaire.

1 Avant d'aller plus avant dans l'analyse du contenu du premier disque, il nous a semblé important de dire quelques mots sur le choix du titre ${ }^{1}$. En effet, il peut paraître incongru de désigner le Yémen comme le cœur de l'Arabie, étant donné la position géographique clairement méridionale que le pays occupe au sein de la Péninsule Arabique. Cependant, parler du «cœur de l'Arabie» traduit ici le fait que le Yémen a de tout temps constitué une terre de haute civilisation, essentiellement sédentaire et urbaine (en opposition au nord plus désertique et abritant de nombreuses populations nomades). Cela est particulièrement vrai en ce qui concerne sa musique : que ce soit les formes modernes de musiques du Golfe, ou les genres plus classiques tels que le şowt (musique savante urbaine) par exemple, l'influence yéménite y est bien assez évidente pour être saisie. Si les Yéménites sont très conscients de l'influence de leur culture musicale sur les pays voisins, il est surtout intéressant de constater l'existence d'une forte conscience chez les 
artistes du Golfe, de ce qu'ils « doivent » au Yémen. Dans cette perspective, le Yémen est bien à considérer comme le « cœur de l'Arabie».

2 La plage 1, intitulée «Ađān $n^{2}$ : premier appel à la prière », met en scène un moment bien connu de quiconque est familier des pays musulmans. Il faut toutefois noter que les voix sont moins mélodieuses et plus "contrôlées» que dans d'autres contrées du monde islamique. Cela s'explique essentiellement par l'appartenance de la majorité des șan'ānī à l'école juridique chiite zaydite, qui représente une tendance plutôt austère de l'islam (notamment ence qui concerne la musique), et qui prône donc une certaine retenue dans l'appel à la prière.

3 Les plages 2, 3, 4 et 6 nous entraînent dans le raffinement et les subtilités du ġinā șan'ānī, le chant de Sanaa. Ce genre musical, savant et urbain, constitue sans doute l'un des plus anciens de la musique yéménite: son existence est en effet attestée par les spécialistes dès le XVIII ${ }^{e}$ siècle. La plage 2 est un très bon exemple de suite qawma. Malheureusement, une erreur est survenue lors de la publication du livret, puisque l'interprète de la pièce en question n'est pas H̄asan 'Awnī al-'Ajamī, mais Muhammad H̄amūd al-H̄āriṫ̄̇̄i. De plus, ce dernier s'accompagne sur le luth oriental ' $\bar{u} d$, et non sur le qanbūs. Il s'ensuit que le disque ne contient aucun exemple musical de Ţarab de Sanaa, ce qui est regrettable. Cette pièce suit le schéma classique de la suite qawma, incluant dans l'ordre un fartaš (prélude musical), une das'a à onze temps, suivie d'une wasţā à 8 temps, et s'achevant sur un sāri', partie la plus rapide également à huit temps. La plage 6 n'est qu'une répétition, plus brève, de l'extrait proposé en plage 2. La plage 3, interprétée par le même chanteur, est un très bon exemple de ginin' șan'ānī accompagné uniquement sur le plateau șahnn, sans adjonction d'instrument mélodique.

4 La pièce suivante est interprétée par Hasan 'Awnī al-'Ajamī, s'accompagnant sur un luth oriental. Al-'Ajamī est également connu pour être l'un des derniers joueurs de Ţarab, dont il est un virtuose reconnu; mais il sait également se servir à merveille du 'ûd. Contrairement à Muhammad al-H̄āritīi, qui est l'un des chanteurs les plus populaires et les plus connus à Sanaa et au Yémen, H̄asan al-'Ajamī n'a jamais pratiqué son art au grand jour. Mais il est l'héritier de l'ancienne tradition du chant de Sanaa. En ce qui concerne la plage 4 , une qawma complète du grand poète du XVI ${ }^{\mathrm{e}}$ siècle, Muhammad Šaraf ad-Dīn, on admirera tout particulièrement la richesse mélodique du jeu de luth de H̄asan al-'Ajamī. Il faut toutefois signaler un petit problème, puisque la traduction du début de la poésie, à l'intérieur du livret, est inexacte. Tel en est le contenu en réalité :

o toi qui rends jaloux la gazelle et le faon

Voici mon âme, rends-moi justice et rejoins-moi (traduction Jean Lambert).

Que ce soit sa version du chant de Sanaa, qui se veut directement issue de l'ancienne tradition, ou celle de Muhammad al-H̄āritīì, peut-être moins classique dans sa forme, nous avons là deux exemples de la grande liberté d'interprétation personnelle que permet le genre. La voix d'al-'Ajamī est ainsi puissante et aiguë et relativement nasillarde, celle d'alH̄āritīi s'exprimant dans un registre plus grave et plus sourd. Les phrasés du luth sont également caractéristiques, et chaque musicien développe sa propre aptitude à exploiter les modes et mélodies du gināà

6 Parallèlement à la tradition profane du chant de Sanaa, un art religieux exclusivement vocal a fleuri: il s'agit du našíd. Du fait de son caractère sacré, toute utilisation d'instrument autre que la voix humaine - seule création exclusivement divine - y est proscrite. On chante le našìd lors de célébrations telles que mariages, circoncisions, décès, 
beaucoup plus rarement dans le contexte de la séance de qāt magyal. La plage 5 nous donne un bon exemple de mašrab, forme de našĩd célébrant l'amour du Prophète sur des thèmes extrêmement variés. La plage 14 est composée d'un autre mašrab, dont l'auteur est le très grand Ahmad Šawqī, surnommé Amīr ǎs-Šu'arā', "prince des poètes ». La première pièce est interprétée par le grand naššād șan'ānī Muhammad Qāsim Zubeida, alors que la seconde a pour chanteur principal un jeune garçon sans doute encore en formation.

$7 \quad$ La plage 9 présente une facette beaucoup plus orchestrale de la musique yéménite, avec un bel extrait de la chanson de Lahj, célèbre région au nord d'Aden. Quant aux plages 7, 8 et 15 , elles proposent trois brefs extraits de musiques du Hadramaout, la grande province de l'est. Le premier est composé de musique populaire chantée, sur un poème de caractère profane ; le deuxième met en scène un joueur de clarinette double mizmār, et le dernier nous emmène à Shibam, pour une récitation nocturne du Coran.

8 Il faut encore signaler quelques petites erreurs dans le commentaire de ce CD. C'est ainsi que la plage 10 présente une pièce jouée à la lyre non pas simsimiyya, mais Ţumbura, dans le registre $d u$ répertoire $d u$ zār (voir ci-dessous); par ailleurs, dans l'index des membranophones en p. 9, il faut lire marfa“ et non " marba», et ce ne sont pas les timbales qui sont frappées avec les mains mais les tambours maasta et marod ${ }^{3}$.

Enfin, l'interruption en plein milieu, de la suite șan'ānī chantée et jouée par H̄asan al-'Ajamī sur le Ţarab, et qui constitue le seul exemple de cet instrument, aurait pu être évitée. Globalement, le film fournit néanmoins un bon aperçu de la diversité des terroirs musicaux du Yémen, tout en en permettant une bonne contextualisation, pas toujours évidente à se représenter pour le néophyte.

Le deuxième $\mathrm{CD}$ est une réédition du seul disque exclusivement dédié aux musiques de la Tihāma yéménite, basses terres bordant la Mer Rouge. Ces enregistrements réalisés par Anderson Bakewell, avaient paru en 1985 sous le titre un brin moins aguicheur de "The Afro-Arabian Crossroad: Music of the Tihāma on the Red Sea " (Lyrichord LLST 7384). Vingt ans plus tard, ils constituent la seule source sonore publiée - à l'exception de quelques pièces éparses enregistrées par Alain Saint-Hilaire en 1973, par la mission Christian Poché en 1975, et par John Levy - donnant à entendre, peu ou prou, l'extraordinaire richesse des répertoires musicaux de la Tihāma.

11 Evoquons tout d'abord la cohérence thématique des 15 plages du disque, enregistrées en $1982^{4}$. L'accent est mis principalement sur les musiques de danse, et en particulier sur les ensembles de percussions muŢabbalin, généralement accompagnés d'une flûte qașaba ou šubbibe. Les plages 7, 11-13 et 15 illustrent l'une des grandes occasions de danser: les pèlerinages annuels ziyāra aux saints, encore relativement fréquents dans cette région sunnite shaféite. Le jeu rythmique de la grande lyre Ţumbura est exemplifié au sud et au nord de la Tihāma - à Zabīd (plage 2 et 6) et dans le Wādi Mawr (plage 10) - ce dernier exemple constituant l'unique enregistrement publié du répertoire de zār Ţumbura (un rituel de possession) dans cette région. Enfin, le cas des musiciens professionnels itinérants est particulièrement bien représenté avec le jeu de la lyre simsimiyya à alHudayda (plage 4 et 8), de la double clarinette mizmār (plage 9) et, plus encore, avec un extrait inespéré de la poésie rythmée de Kahtal (plage 14), dont il ne reste aujourd'hui que de très mauvais enregistrements sur cassettes, et qui témoigne de la singulière originalité du répertoire ĥ̀ōāmi. 
12 Le parti pris de présenter les musiques de danse (et éventuellement de «transe », bien que le terme soit discutable) est loin d'être représentatif du large corpus poético-musical d'une région à la géographie sociale complexe. Ainsi, à l'exception de la plage 14, les différents genres de la poésie dialectale chantée échappent, dans une large mesure, à la portée du présent $C D$.

Dans les notes du livret, Anderson Backwell fait à juste titre le lien entre le jeu des percussions en Tihāma et le statut «moralement douteux » des Aĥ̀ām (ou Akhdām),une minorité d'origine prétendument africaine, indissociablement liée au métier de percussionniste, considéré comme une activité « honteuse » $\left(z a^{a} b \vec{a}^{\prime}\right)$. Les flûtes et les lyres, par contre, sont également jouées par d'autres catégories sociales, esclaves ('abìd) - un statut aujourd'hui disparu - ou hommes de tribu ('arab) pour les flûtes, mais à notre connaissance jamais par des femmes.

14 Les plages 1, 3 et 5 illustrent le jeu polyrythmique des ensembles de percussions et flûte à Zabīd ou dans les environs. Les orchestres sont généralement composés d'une flûte, qaș $a b a$ ou šubbibe (et non shabbäba, p. 9), et de quatre membranophones: deux Ţabl (orthographié Ţabla dans le livret), répartis en wosţa' (basse) et marā̄̌ (dynamique), et deux timbales en terre cuite, le grand marfa' (photo p. 2) et le petit dumdumē.

On appréciera l'excellente qualité dynamique de ces prises in situ dont l'atmosphère fiévreuse est bien retransmise, y compris dans l'effet de contrepoint rythmique produit par les claquements de mains de l'assistance (plage 1). Quant à la plage 6, dont on goûtera particulièrement le jeu tonique de la qașaba et la répétition de motifs superposés, on doutera cependant de son appellation de habāš (p. 13), qui pourrait avoir été rapportée à Bakewell dans le sens du terme péjoratif $\mathrm{d}^{\text {' } ~ " E t h i o p i e n s ~ » ~ q u a l i f i a n t ~ l e s ~ A k h d a ̄ m, ~ o u ~}$ encore d'une devise commune au répertoire du zăr, mais qui ne correspond pas à la présente pièce.

Le répertoire du zār tihāmi est ici représenté uniquement par des ensembles polyrythmiques, lyre Ţumbūra et tambours, ou des soli de Ţumbūra (écrite Ţumbara dans le livret). Il convient de noter que, à Zabīd (plage 2 et 6) et dans le Sud de la Tihāma en général, le zār Ţumbura semble avoir eu dans le passé une place marginale dans la pratique du zār tihāmi, et qu'il est aujourd'hui pour ainsi dire inexistant, suite à l'émigration des familles d'adeptes et des grands joueurs de Ţumbura.

17 Ainsi, 'Umar Turaybī (plages 2 et 6 ; c'est bien lui également en plage 12 du CD précédent ! ) ne pratique plus désormais son jeu que comme un hobby, hors des séances rituelles dont est tiré son répertoire. Les motifs musicaux, inlassablement répétés, soutenus par une rythmique binaire ou ternaire, sont soulignés par de courtes phrases chantées ou même de simples onomatopées (plage 2, nommée Lambuna dans le livret, certainement une confusion entre lēwa et Ţumbura). Cette caractéristique de "distorsion" langagière se retrouve ailleurs dans la poésie chantée en Tihāma, par exemple en plage 8 dans le cas d'un joueur de simsimyya, qui reproduit ici une mélodie d'un film indien agrémenté de paroles « façon » hindi !

18 La plage 10 révèle une autre pièce du répertoire du zār Ţumbura, celui du Wādi Māwr, un style qui a essaimé dans tout le nord de la Tihāma jusqu'à Hodaydah. La lyre Ţumbura est accompagnée du grand tambour madiff, de la timbale miškal et du tambourin sā̈fa. La prise est cette fois in situ, et le chant répond en écho à la « voix » lancinante de la ţumbura 

danses šāmī pour les quatre premières lors des processions qui ont lieu à l'occasion des pèlerinages annuels des saints en Tihāma. La séquence rythmique taH̄dìra présentée à la plage 15 évoque la préparation, l'échauffement. Elle est assez proche du rythme joué en plage 9 (il faut lire šara $\bar{H}$ et non sharha), et entraîne des danses acrobatiques ou des danses de force quwwa, dont celles du registre hodāmī pratiqué quasi-exclusivement par les Akhdām.

Des trois plages consacrées aux musiciens professionnels itinérants, on retiendra, outre l'emploi de la simsimiyya dans le registre libre et improvisé de l'adaptation des chansons populaires ou "folkloriques» (plages 4 et 8), la grande liberté [d'improvisation et] d'expression dans l'exécution de ces pièces. Quant à la plage 14, elle présente Kahtel et Kubba, deux musiciens aveugles aujourd'hui décédés, qui ont produit, accompagnés l'un d'une qașaba et l'autre d'un bidon percuté, une poésie libre, qui s'exprime dans un style confiné à l'autodérision et à la parodie.

Au final, les quinze plages de ce $C D$ s'avèrent passionnantes à plusieurs titres. D'abord parce qu'elles présentent un matériel inédit, qui dévoile quelque peu la complexité syncrétique et la profusion des particularismes des répertoires rythmiques, mélodiques et vocaux en Tihāma. Le plaisir de découvrir des pièces déjà anciennes est doublé de l'excellence des prises de son, qui restituent bien la dynamique de l'instrumentation et des voix, alors que presque toutes ont été enregistrées in situ, au milieu d'une foule ou lors d'un rituel de zār privé laissant généralement peu d'opportunité à l'enregistrement. Enfin, les photos d'Alain Saint-Hilaire sont toujours superbes, les choix du fade out s'avèrent judicieux, et les notes du livret sont dans l'ensemble fouillées.

Ainsi, si la notion de conservatoire ou de «tradition » est justifiable dans l'appréhension des musiques savantes urbaines, elle n'est pas non plus inutile pour penser ces répertoires moins fixés, plus mouvants peut-être, mais pour le moins sujets à une " érosion " croissante, voire à une disparition à court terme. En effet, la percée de la télévision et de la radio, la transformation de l'idéologie religieuse et les bouleversements économiques et sociaux remettent aujourd'hui en question, avec l'atomisation sociale, la fragile pérennité d'un nombre non négligeable de ces répertoires.

\section{NOTES}

1. Nous avons résolument choisi d'éclairer et de situer ces disques à la lumière de nos terrains actuels : Samir Mokrani travaille sur la préservation du chant de Sanaa ; Luc Weissenberg sur les milieux du zār et la littérature orale à Zabīd.

2. Dans le système de translittération adopté ici, š se lit «sh», ĥ se lit «kh»; đ se lit « dh », etc. Certains termes dialectaux sont transcrits tels quels.

3. Signalons l'existence du film Yémen: Musique du cœur de l'Arabie, réalisé par Layth Abdulamir et Henri Lecomte $\left(2000,52^{\prime}\right)$, qui fournit un sympathique complément audiovisuel à ce CD.

4. Il semble que les archives complètes de cette mission soient déposées à la British Library. 